\title{
Stable incremental deformation of a strip to high strain.
}

\author{
A. Hadoush ${ }^{1,2, a}$, A.H. van den Boogaard ${ }^{2, b}$, J. Huétink ${ }^{2, c}$ \\ ${ }^{1}$ Netherlands Institute for Metals Research, P.O. Box 5008, \\ 2600 GA Delft, The Netherlands. \\ ${ }^{2}$ Faculty of Engineering Technology, University of Twente, P.O. Box 217, \\ 7500 AE Enschede, The Netherlands. \\ aa.hadoush@ctw.utwente.nl, ${ }^{\mathrm{b} a}$ a.h.vandenboogaard@ctw.utwente.nl, \\ cj.huetink@ctw.utwente.nl
}

Keywords: Incremental forming; Bending; Stretching; Forming limit

\begin{abstract}
This paper presents the effect of combined stretching and bending on the achieved strain in incremental sheet forming ISF. A simple two dimensional model of strip undergoing stretching and travelling three point bending in cyclic form is used. The numerical model presents the effect of the ratio of stretching velocity to roll-set speed on the achieved strain and its distribution.
\end{abstract}

\section{Introduction}

Several aspects of ISF process are not clearly understood. How does the strain achieve higher limit values far beyond the FLC? How is it possible to manufacture material with low or zero formability [1]? ISF is commonly considered as plane strain stretching of the sheet, and is modelled by the sine law. This model is not able to explain the thickness distribution of the sheet nor the achieved strain, since it does not consider bending effects.

Stretch under three point bending is also applied in the tension levelling process, which is used to straighten sheets [2]. It is hard to perform cyclic tests on sheet metal completely equivalent to ISF, since it includes several complex aspects e.g. bending with double curvature. Introducing a simple model to investigate the influence of cycling in sheet forming is commonly used $[3,4]$. A new approach is defined to simplify the ISF process by a strip, which experience stretching and cyclic three point bending. Our approach differs from tension levelling by including the cyclic effect

\section{Models and process descriptions.}

The model presents a strip of $1 \mathrm{~mm}$ thickness and $110 \mathrm{~mm}$ length, without imperfection. It is stretched by controlled displacement at both ends, and bent in a cyclic form by a roll-set as shown in Fig. 1.

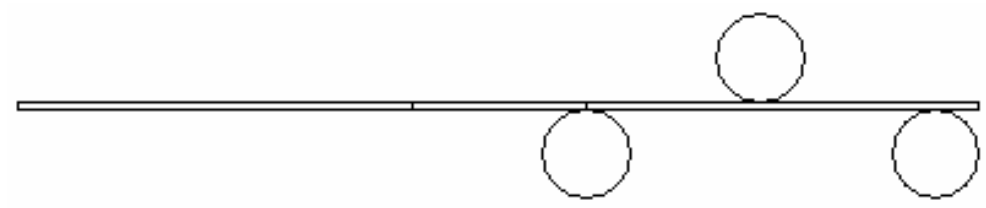

Fig. 1. The strip and the roll-set at its original position.

The strip is stretched at the ends enough to initiate plastic elongation. The ends are held, then the central roll deforms the strip by its motion perpendicular to the longitudinal axis of the strip, this motion by itself is also enough to cause plastic deformation. The movement of the roll-set from one side to the other causes the cyclic effect. The strip is stretched simultaneously with the 
cyclic movement of the roll-set. Each roll is $10 \mathrm{~mm}$ in diameter, the centre of the right and left roll w.r.t. the central roll, are $20 \mathrm{~mm}$ apart from the central roll. A special part of the strip experiences more cyclic effect than the rest of the strip, this part is located between the left roll at its original position and the right roll when the set totally moved to the other side of the strip. In other words, the $20 \mathrm{~mm}$ of the strip at the centre is of interest; it will be referenced as the central deformation zone. In physical experiments, this part can be made much larger. Here only a small part is modelled to reduce the computing time, since a larger part leads to more numerical elements for the simulation and it needs longer stroke to cover.

The deformation of the strip is modelled as plane strain, quasi static force equilibrium. Von Mises yield criterion is used; the elastoplastic material behaviour is governed by Nadai hardening as follows in Eq. 1

$$
\sigma=500(\varepsilon+0.00243)^{0.2}
$$

Where $\sigma$ and $\varepsilon$ are the stress and the plastic strain, respectively. The initial yield stress is $150 \mathrm{MPa}$. The material model is representative of mild steel.

Numerically, finite element simulations are done using the in-house implicit finite element code Dieka. The discretization of the geometry is done by linear quadrilateral element, four element in thickness and 550 along the length. At the beginning of the process, the strip is stretched 0.153 $\mathrm{mm}$ at each end, $1 \mathrm{~mm}$ of central roll perpendicular motion causing bending, followed by cyclic axial motion of the roll-set of $60 \mathrm{~mm}$, as stroke is performed, no perpendicular motion.

\section{Validation}

Combined bending and stretching is one of the reasons of achieving higher strain far beyond the FLC. Because of the resultant tension stress of bending combined with stretching, part of the strip fibers tend to deform in exaggerate way if it is not connected to the compressed fibers, which pull the elongated fibers back; resulting in a stabilized material deformation. Initial calculations show that, as soon as the bending causes no compressed fibers because of hardening, the material fails under tension, after achieving higher strain.

The evaluation of failure is done numerically, since an analytical model is not available at the moment. The following analysis and figures are related to the central deformation zone, since it experiences more cycling than the rest of the strip. Now, the nodal values of displacement in the longitudinal direction of the midplane are taken as source of data, hence the neutral location is changed regarding the bending from one side to the other side of the midplane. The nodal values of displacement are plotted versus the incremental step number as shown in Fig. 2, as soon as a significant divergence between any adjacent nodes is detected, material is failed, and all previous increments are considered as stable deformation of the material. Using this approach the need of imperfection is investigated.

The failure of the strip has to be in the central deformation zone, at the part, which experienced the most severe deformation history. But, does the model need an imperfection in the numerical model to trigger a localised deformation? To investigate that, a small geometrical imperfection is initiated by reducing the thickness of the strip by $0.2 \%$ at specific location at the central deformation zone, using DC10 the simulation is done for a geometrically imperfect model and compared to geometrically perfect model. Both models with and without imperfection failed at 
the same cycle, the strain distribution for both model is plotted in Fig. 3. The strain reaches almost the same level in both models, the imperfection model triggers another localisation at the imperfection location. It appears that the imperfection does not influence the achieved strain, to clarify this point, the difference in strain for the last stable cycle is plotted in Fig. 4.

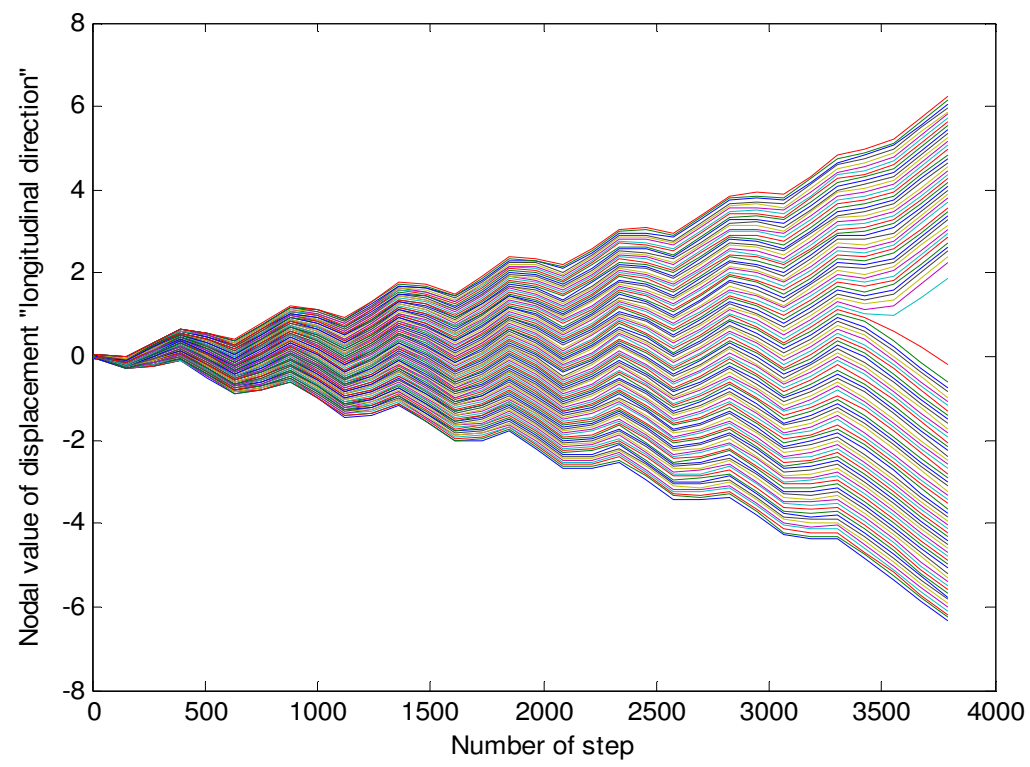

Fig. 2. Failure detection technique.

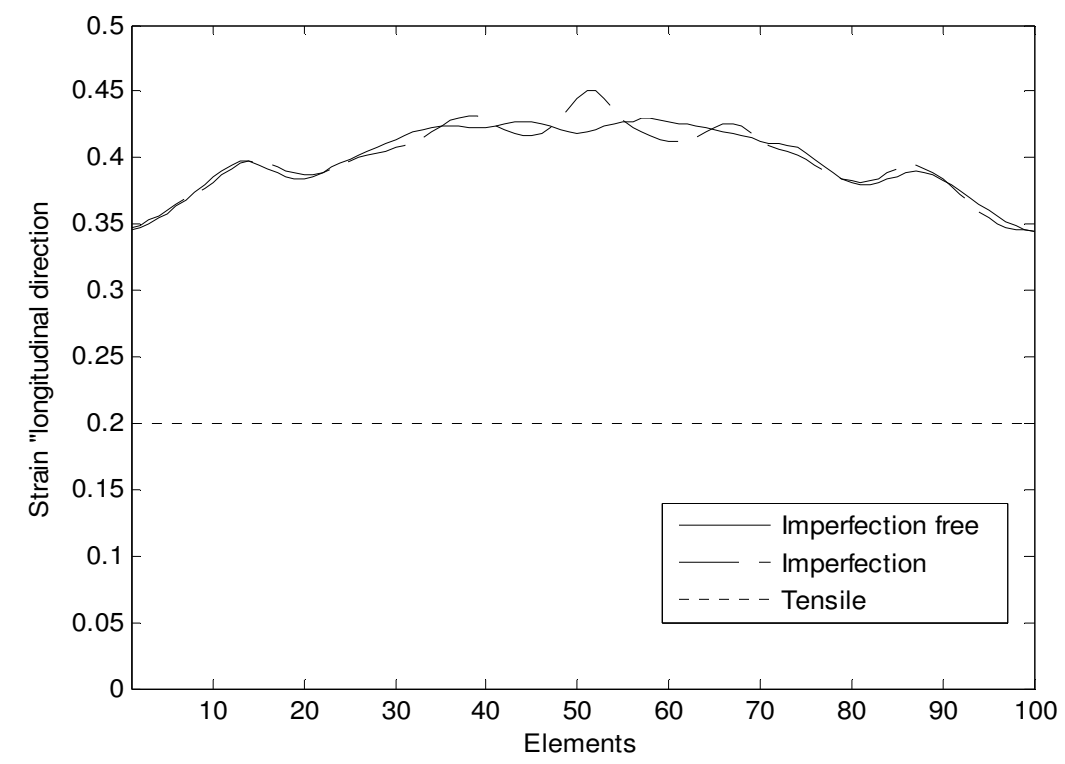

Fig. 3. Strain distribution of an imperfect and imperfection free model.

The maximum difference is 0.03 , located at element number 51 , which is at the imperfection. The difference in strain can be explained by the reduction of area at the imperfection location. The imperfection hardly reduces the achieved strain by localising the deformation. Indeed, as shown in Fig. 3 the strain distribution for both models is non uniform, as consequent there is no need for imperfection. 


\section{Influence of stretching velocity}

The ratio of stretching velocity to roll-set speed DC is investigated. The initial ratio is $0.0016, \mathrm{DC}$ is scaled by a factor 1,5 and 10 for each set of simulation. The strain distributions along the central deformation zone, for the last stable cycle of the three scaling factors are plotted in Fig. 5. The increase of stretching at the ends leads to lower achieved strain, but on the other hand the strain looks more uniform, but it is not completely uniform, as clearly observed in cases DC10 and DC5. The total number of completed cycles differs from one scaling factor to another, it is 85 , 16 and 7 for DC1, DC5 and DC10 respectively, which means that the material sustains more cycles with less stretching.

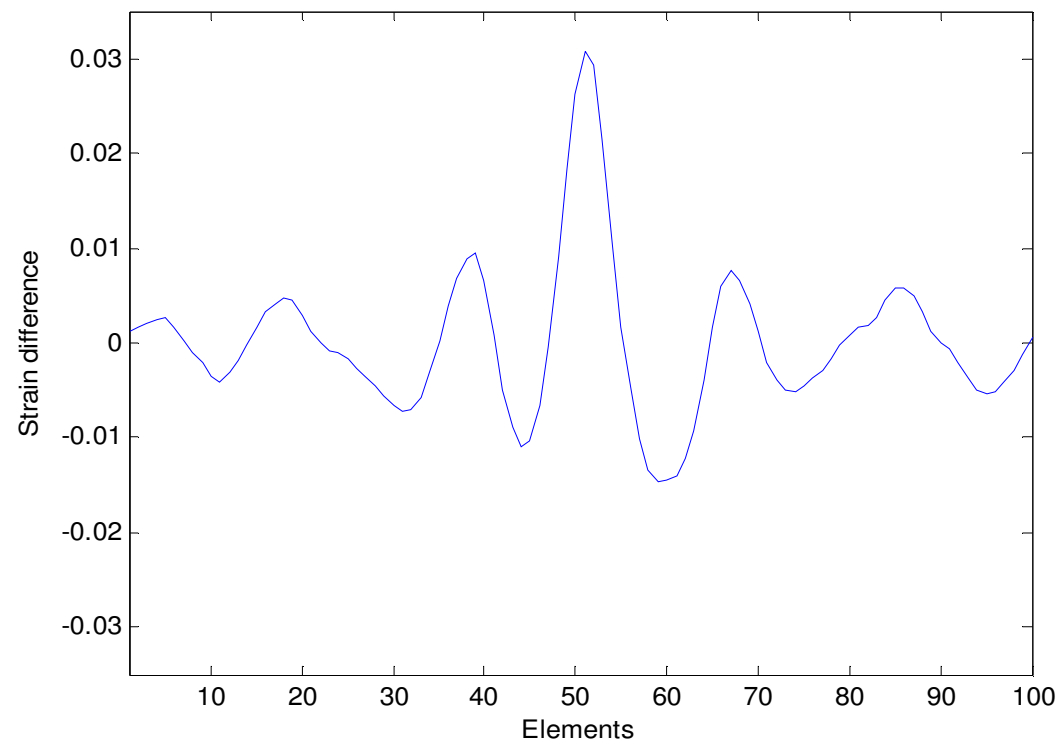

Fig. 4. Strain difference between models with and without imperfection.

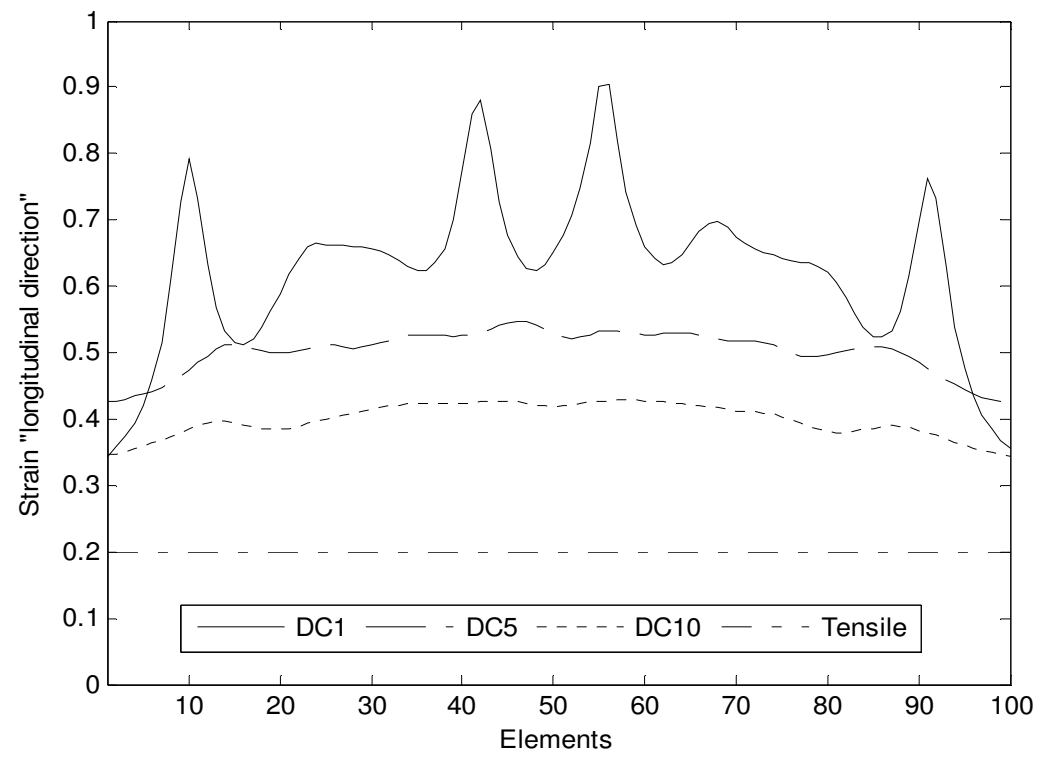

Fig. 5. Strain distribution for stable material deformation for different scaling factor of DC. 
A non-symmetric distribution of the strain along the central deformation zone is expected, since the process itself is direction dependent. A uniform strain distribution along the strip is preformed by stretching at the beginning of the process, but as soon as the central roll starts deforming the strip by its vertical motion a non-symmetric bending load is present because of the geometry. During the process, the motion of the roll-set stretches part of the strip, which is located at the opposite side of the motion more than the other part, as a consequence of this combination of stretching and bending, a non-symmetric stress distribution is present, which draws the material to different levels of hardening. In other words, for each increment of deformation, each part of the material has its unique history of hardening.

Based on the special characteristic of the process, another interesting phenomenon is realised, which will be mentioned as multi-localisation. Multi-localisation is observed in the strain distribution of DC1, which is shown in Fig. 5. Even if localisation starts at a specific location at the central deformation zone, the rest of the central deformation zone still deforms. Because of the rollset cyclic movement, that shifts the maximum stress from one location to another. This incremental movement acts like a switch, based on this movement part of the central deformation zone will deform more than the rest. Regardless of being a localised region or not, the part which experiences the maximum stress state does deform. That may create, based on the load combination another localised deformation. Multi-localisation is developed at each cycle of the process, it does not appear suddenly in the strain distribution, the development progress of multi-localisation for the DC1 simulation is plotted in Fig. 6. It clearly shows that also in the non localised regions, the strain increases with further cycling.

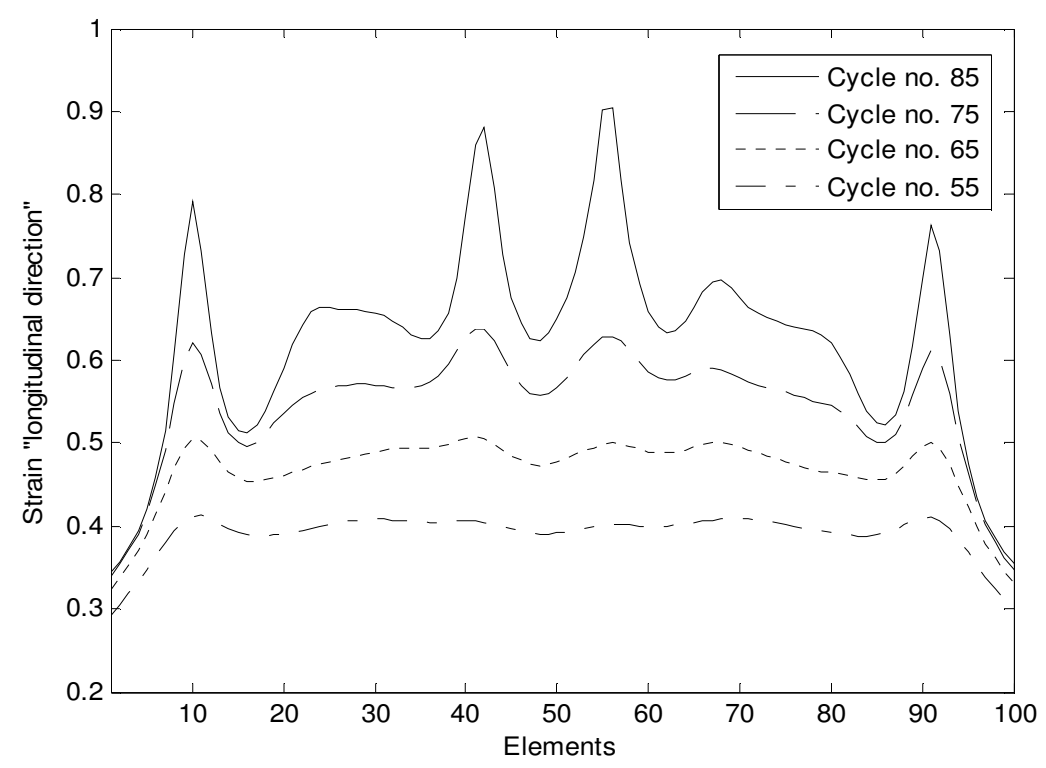

Fig. 6. The development of multi-localisation during the DC1 process.

\section{Conclusion}

The combined load of stretching and cyclic bending together with the directional dependency of the process are the key factors of the achieved strain and its unique distribution. For better understanding, the deformation in the incremental forming process should be investigated for all intermediate stages of the process not only at the end of each cycle. Based on the ability of the model, an extension for three dimensional space will clarify some of incremental sheet forming's secrets and create better guide lines than using a FLC. 


\section{References}

[1] W.C. Emmens, Water jet forming of steel beverage cans, International Journal of Machine Tools \& manufacture 46, 2006, pp 1243-1247.

[2] F. Yoshida and M. Urabe, FE simulation of tension levelling process for clad sheet metals, Advanced Technology of Plasticity, vol. 2, Proceeding of the $6^{\text {th }}$ ICTP, 1999, pp. 1107-1112.

[3] L.W. Meyer, C. Gahlert and F. Hahn, Influence of an incremental deformation on material behaviour and forming limit of Aluminium A199,5 and QT-Steel 42CrMo4, Advanced Materials Research, vol. 6-8, 2005, pp. 417-424.

[4] F. Yoshida, T. Uemori and K. Fujiwara, Elastic-plastic behaviour of steel sheets under in-plane cyclic tension-compression at large strain, International Journal of Plasticity, vol. 18, 2002, pp.633659. 